\title{
End-to-end Mars entry, descent, and landing modeling and simulations for Tianwen-1 guidance, navigation, and control system
}

\author{
Chao $\mathbf{X u}^{1,2}$, Xiangyu Huang ${ }^{1,2}(\bowtie)$, Minwen $\mathbf{G u o}^{1,2}$, Maodeng $\mathbf{L i}^{1,2}$, Jinchang $\mathbf{H u}^{1,2}$, \\ and Xiaolei Wang ${ }^{1}$
}

1. Beijing Institute of Control Engineering, Beijing 100094, China

2. National Key Laboratory of Science and Technology on Space Intelligent Control, Beijing 100094, China

\begin{abstract}
On May 15, 2021, the Tianwen-1 lander successfully touched down on the surface of Mars. To ensure the success of the landing mission, an end-to-end Mars entry, descent, and landing (EDL) simulator is developed to assess the guidance, navigation, and control (GNC) system performance, and determine the critical operation and lander parameters. The high-fidelity models of the Mars atmosphere, parachute, and lander system that are incorporated into the simulator are described. Using the developed simulator, simulations of the Tianwen-1 lander EDL are performed. The results indicate that the simulator is valid, and the GNC system of the Tianwen-1 lander exhibits excellent performance.
\end{abstract}

\author{
KEYWORDS \\ Mars entry, descent, and \\ landing (EDL) \\ modeling and simulation \\ Tianwen-1 lander \\ guidance, navigation, and \\ control (GNC) \\ Research Article \\ Received: 16 September 2021 \\ Accepted: 9 October 2021 \\ (C) The Author(s) 2022
}

\section{Introduction}

The Tianwen-1 mission to Mars was launched on July 23, 2020. On May 15, 2021, after approximately 9 min of the entry, descent, and landing (EDL) phase, the Tianwen-1 lander successfully touched down on the surface of Mars. The Mars EDL phase began at the atmospheric interface at a radial altitude of $125 \mathrm{~km}$ and ended with a surface touchdown $[1,2]$. This paper describes the EDL modeling and simulation of the Tianwen-1 EDL mission.

The Tianwen-1 EDL modeling and simulation are aimed at designing and validating the lander guidance, navigation, and control (GNC) system, which ensures accuracy and safe touchdown. In the EDL phase, the Tianwen-1 lander sequentially performs trim wing deployment, parachute deployment, heatshield ejection, and backshell separation in the highly uncertain Mars environment [3]. Thus, the dynamics of the EDL phase are complicated and involve multiple bodies with significant interactions [4]. To accurately model the dynamics of the EDL phase, we develop an end-to-end Mars EDL simulation tool for the Tianwen-1 GNC system, including high-fidelity models of the Mars atmosphere, parachute, and lander system. This simulation tool can be used to assess the GNC system performance and determine the critical mission and lander parameters.

The National Aeronautics Space Administration (NASA) developed two primary Mars EDL simulation tools, based on NASA LaRC's program to optimize simulated trajectories II $[5,6]$ and NASA JPL's dynamics simulator for entry, descent, and surface landing $[7,8]$. Based on the two simulators, NASA performed Mars EDL simulations for the Mars Phoenix and Mars Science Laboratory and successfully landed them on the Mars surface [9, 10]. Several of our simulator models, including the Mars atmosphere environment and parachute dynamics, have been developed with reference to the foregoing Mars EDL flight experiences [11-13], with modifications introduced to the Tianwen-1 lander. To satisfy the requirements of parachute deployment, the trim wing of the Tianwen-1 lander is deployed at a specified velocity to secure a zero trim angle of attack that differs from previous Mars landing missions. Accordingly, the dynamics of the Tianwen-1 lander with the deployed trim wing have been modeled in our 


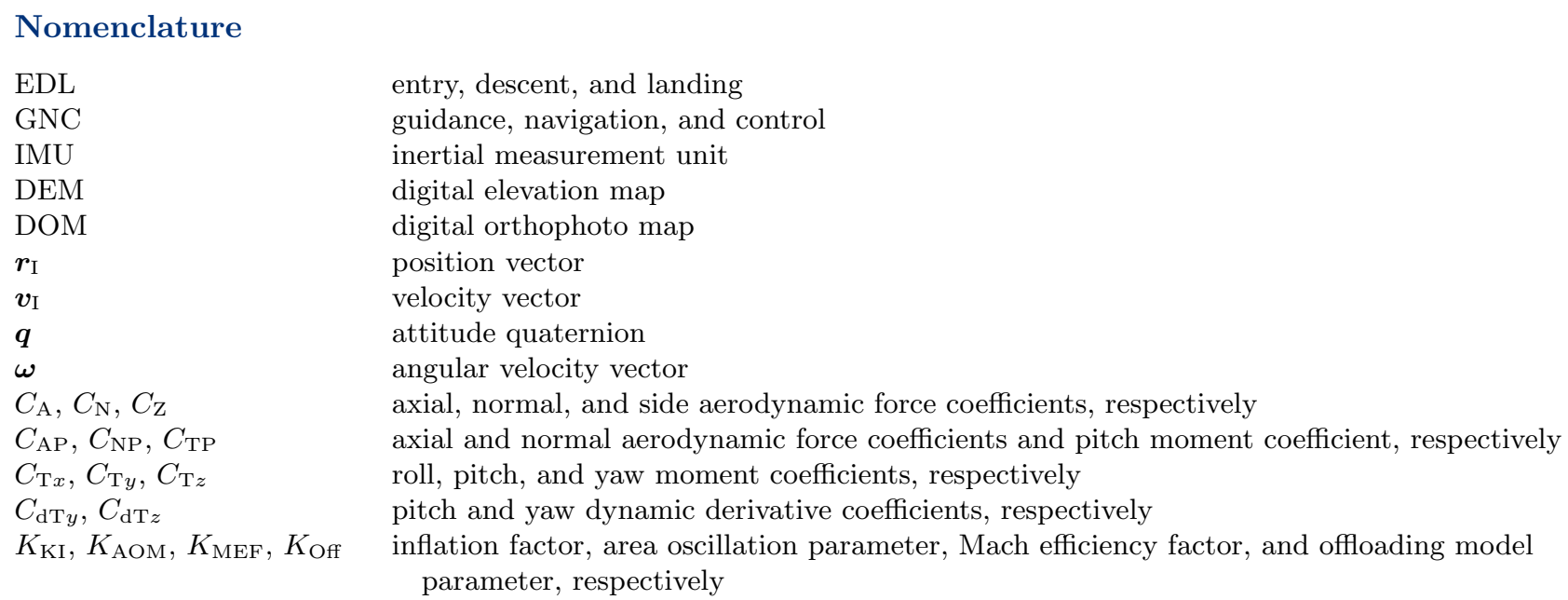

EDL

GNC

IMU

DEM

DOM

$r_{\mathrm{I}}$

$v_{\mathrm{I}}$

$q$

$\omega$

$C_{\mathrm{A}}, C_{\mathrm{N}}, C_{\mathrm{Z}}$

$C_{\mathrm{AP}}, C_{\mathrm{NP}}, C_{\mathrm{TP}}$

$C_{\mathrm{T} x}, C_{\mathrm{T} y}, C_{\mathrm{T} z}$

$C_{\mathrm{dT} y}, C_{\mathrm{dT} z}$

$K_{\mathrm{KI}}, K_{\mathrm{AOM}}, K_{\mathrm{MEF}}, K_{\mathrm{Off}}$

entry, descent, and landing

guidance, navigation, and control

inertial measurement unit

digital elevation map

digital orthophoto map

position vector

velocity vector

attitude quaternion

angular velocity vector

axial, normal, and side aerodynamic force coefficients, respectively

axial and normal aerodynamic force coefficients and pitch moment coefficient, respectively

roll, pitch, and yaw moment coefficients, respectively

pitch and yaw dynamic derivative coefficients, respectively

inflation factor, area oscillation parameter, Mach efficiency factor, and offloading model

parameter, respectively

simulator. Moreover, we have developed interpolation methods for accurately simulating the aerodynamics, parachute dynamics, and dynamics processes of trim wing deployment, parachute deployment, heatshield injection, and backshell separation. We have also adopted multi-threading and parallel processing technologies to design our simulator. Based on these methods, our simulator simplifies the calculation of the dynamics and enables the implementation of a Monte Carlo simulation with tens of thousands of runs over a brief period.

Compared with the extensive research conducted by the United States on Mars EDL modeling and simulation, Chinese scholars have focused more on the GNC technology associated with the Mars EDL [14, 15]. Although certain studies have been focused on parachute dynamics [16-18], the frameworks are mainly theoretical and not suitable for engineering applications. As described in this paper, we update the existing Mars atmosphere environment and parachute dynamics model and develop several models to construct a complete Mars EDL simulator for the Tianwen-1 lander. With the above equipment, several simulations for evaluating the capability and performance of the Tianwen-1 lander GNC system are performed.

\section{Tianwen-1 EDL mission overview}

\subsection{Tianwen-1 lander configuration}

The Tianwen-1 lander consists of heatshield, backshell, and landing platform carrying a rover, as shown in Fig. 1. The backshell contains a trim wing and a parachute system, which are sequentially deployed

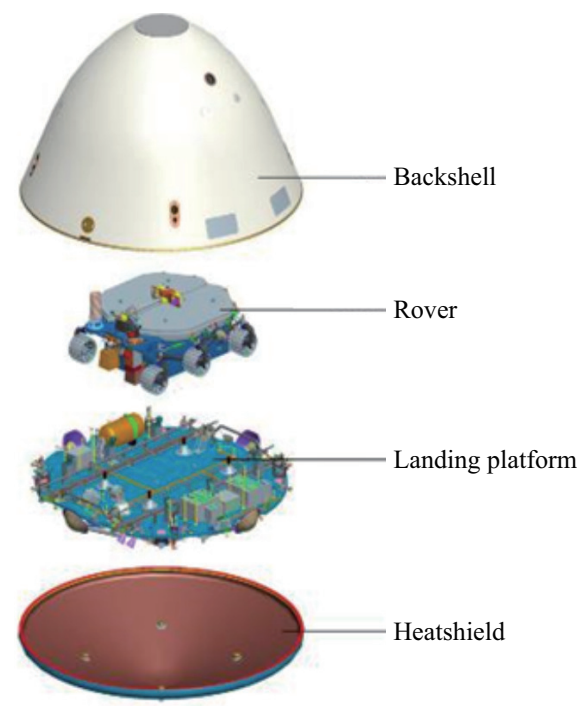

Fig. 1 Tianwen-1 lander configuration. Reproduced with permission from Ref. [3], (C) Xiangyu Huang et al. Exclusive Licensee Beijing Institute of Technology Press.

at scheduled periods. The lander is equipped with navigation sensors, including an inertial measurement unit (IMU), a multibeam radar, a Mars imaging sensor, actuators (including 26 attitude control thrusters), and a variable-thrust main engine. The IMU includes gyros and accelerometers, which measure the three-axis attitude angular velocity and nongravitational acceleration of the lander. The multibeam radar measures the distance to the ground and velocity along each beam direction. The Mars imaging sensors include optical imaging sensors and three-dimensional (3D) laser imaging sensors, which are used to obtain images of the Mars surface and identify safe landing sites while avoiding hazards. 


\subsection{Tianwen-1 EDL sequence phase and operation}

The EDL sequence profile of the Tianwen-1 lander, which consists of atmospheric entry, parachute descent, and landing phases, is shown in Fig. 2. The Tianwen-1 EDL mission is initiated upon atmospheric entry, which is regarded to occur at an altitude of approximately $125 \mathrm{~km}$ with an entry inertial velocity of $4.8 \mathrm{~km} / \mathrm{s}$. When the lander decelerates to 2.8 Mach, the trim wing is deployed, and the angle of attack is trimmed to zero, which is suitable for parachute deployment. When the lander decelerates to $1.8 \mathrm{March}$, the parachute is ejected from the rear of the backshell, and the parachute descent phase is initiated. In this phase, the heatshield is jettisoned at a specific time after the parachute deployment, and the lander legs are deployed. Once the lander legs are deployed, the landing radar begins to measure the vehicle altitude and velocity with respect to the Mars surface. The parachute descent continues until the navigated altitude and velocity satisfy the requirements of powered descent. Next, the lander releases the backshell and ignites the main engine, and the landing phase begins. In this phase, the lander performs velocity reduction, backshell evasion maneuvers, and hazard avoidance. Finally, it performs soft touchdown on the selected landing site.

The Tianwen-1 EDL simulation must model all the
EDL phases and operations, which are triggered by the GNC software included in the simulation.

\section{Tianwen-1 EDL end-to-end simulator}

The architecture of the simulator is illustrated in Fig. 3. The simulator includes six modules, specifically those associated with the Mars environment, dynamics, sensors, Mars terrain, onboard GNC software, and actuator. The dynamics module calculates the aerodynamic force and torque according to the temperature, density, and wind at the current position in the Mars environment. Combined with the thrust effect, the dynamics module can solve the multibody orbit and attitude dynamics problem. According to the orbit and attitude dynamics information, the sensor module simulates the measurements of various navigation sensors. In particular, the radar and imaging sensors require Mars terrain data to simulate their measurements. Thus, the simulator contains a Mars terrain module, which consists of the digital elevation and orthophoto maps of the landing area. After the onboard GNC software receives the sensor measurements, the GNC algorithm estimates the state of the lander and calculates the guidance and control commands according to the estimated states. Subsequently, the guidance and control commands are transformed into thruster pulse width commands, which are transmitted to the actuator module. The thrusters in the actuator

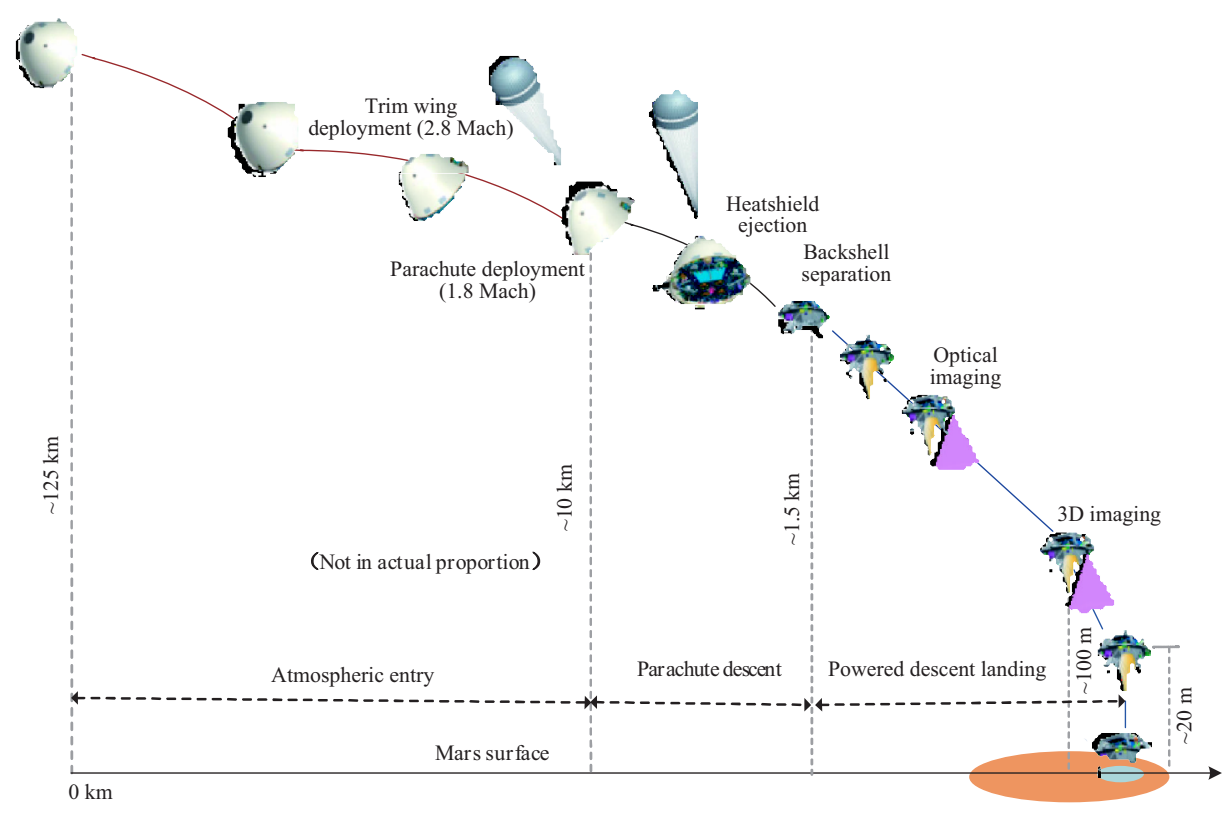

Fig. 2 Tianwen-1 lander sequence phases and operations.

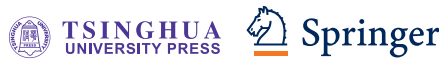




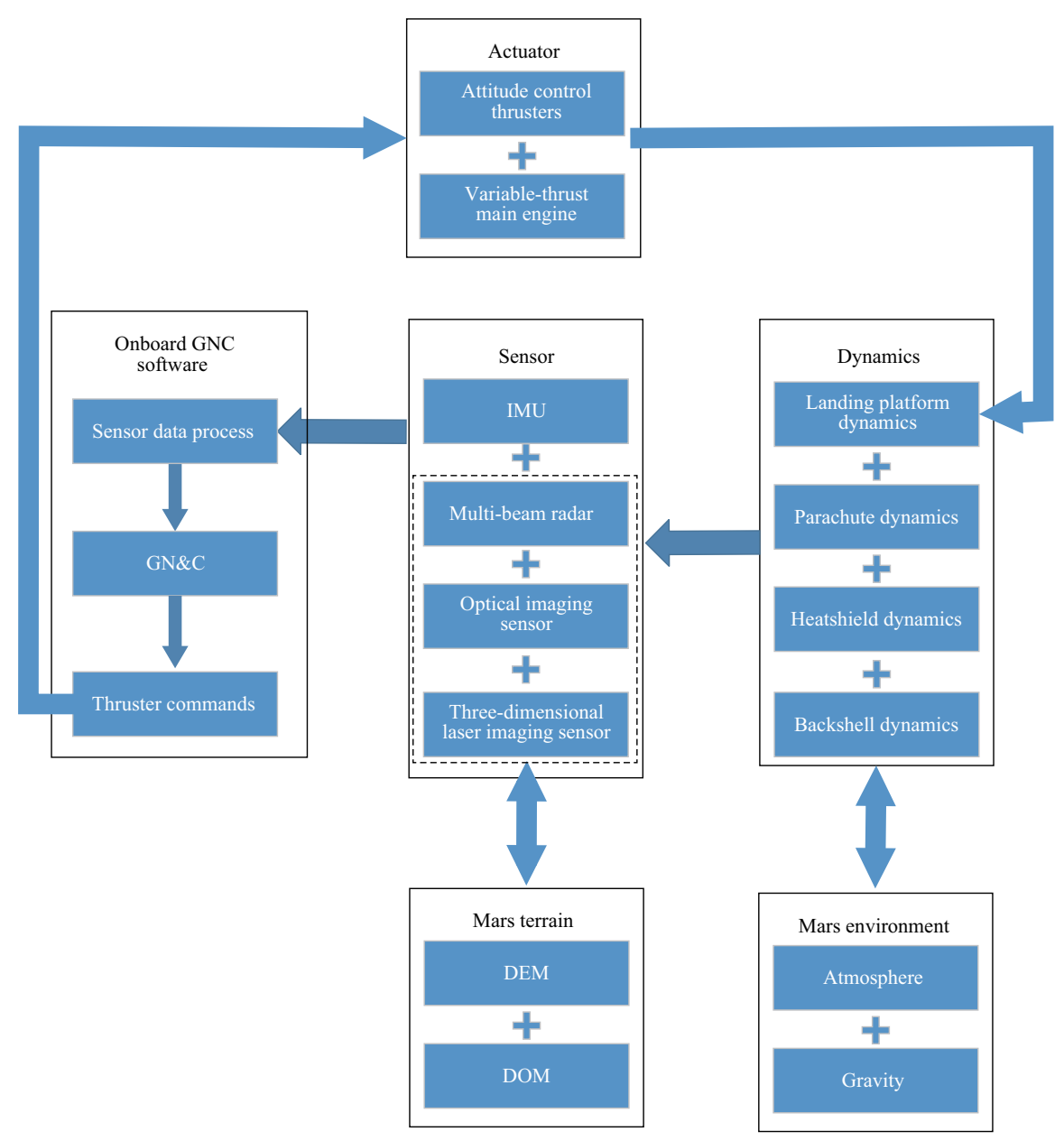

Fig. 3 Architecture of Tianwen-1 EDL simulator.

module generate the corresponding force according to these commands. Based on these aspects, a closed-loop simulator is established, and an end-to-end simulation for the Mars EDL can be performed. The simulator can be used to design and tune the Tianwen-1 lander and EDL operation parameters, develop and validate the GNC algorithm, and implement Monte Carlo analyses of the GNC performance, including precision landing, touchdown velocity, and propellant consumption.

\section{Modeling}

\subsection{Modeling overview}

The six modules described in Section 3 are modeled in the developed Tianwen-1 EDL simulator. This study is focused on the modeling of the Mars environment, multibody dynamics, and GNC algorithm, which are unique and crucial for the Tianwen-1 EDL mission.

As described in Section 2, the Mars EDL phase consists of atmospheric entry, parachute descent, and landing phases. In the atmospheric entry phase, the Tianwen-1 lander with the backshell, heatshield, and packed parachute is treated as a rigid body (known as the entry capsule). The entry capsule is subjected to gravity, aerodynamic force, and engine thrust. Modeling the aerodynamic force is a key element of the Tianwen-1 EDL simulator because knowledge regarding the Mars atmospheric environment is insufficient; thus, its atmosphere must be regarded as highly uncertain. Another key problem is the modeling of parachute dynamics involving the phases of parachute ejection, parachute line stretching, parachute inflation, and stabilized descent. In these phases, for simplicity, the parachute and lander are modeled as two rigid bodies connected by parachute lines. Once the heatshield is ejected, the heatshield dynamics are modeled and solved to verify whether the heatshield is safely separated. The 
influence of heatshield separation is evaluated based on its impact on the radar beam. Similarly, after backshell separation, the parachute force is exerted on the backshell by parachute lines, and the backshell dynamics are modeled and solved to validate and evaluate the parachute-backshell avoidance. The GNC module provides accurate and reliable state estimates and key operation triggers as well as implements accurate and reliable trajectory and attitude controls. In this context, it is a core module validated by the simulator.

\subsection{Tianwen-1 lander dynamics and modeling}

The lander dynamics are modeled using two reference frames: the Mars-centered J2000 inertial frame and lander body frame. The lander dynamics are described in the Mars-centered J2000 inertial frame as

$$
\left\{\begin{array}{l}
\dot{\boldsymbol{r}}_{\mathrm{I}}=\boldsymbol{v}_{\mathrm{I}} \\
\dot{\boldsymbol{v}}_{\mathrm{I}}=\boldsymbol{g}_{\mathrm{mI}}+\boldsymbol{a}_{\mathrm{thr}}+\boldsymbol{a}_{\mathrm{atm}}+\boldsymbol{a}_{\mathrm{para}}+\boldsymbol{a}_{\mathrm{hea}} \\
\dot{\boldsymbol{q}}=\frac{1}{2}\left[\begin{array}{cr}
-\tilde{\boldsymbol{\omega}} & \boldsymbol{\omega} \\
-\boldsymbol{\omega}^{\mathrm{T}} & 0
\end{array}\right] \boldsymbol{q} \\
\boldsymbol{I} \dot{\boldsymbol{\omega}}+\dot{\boldsymbol{I}} \boldsymbol{\omega}+\tilde{\boldsymbol{\omega}} \boldsymbol{I} \boldsymbol{\omega}=\boldsymbol{T}_{\mathrm{thr}}+\boldsymbol{T}_{\mathrm{atm}}+\boldsymbol{T}_{\mathrm{para}}+\boldsymbol{T}_{\text {hea }}
\end{array}\right.
$$

where $\boldsymbol{r}_{\text {I }}$ and $\boldsymbol{v}_{\text {I }}$ are the lander position and velocity vectors in the Mars-centered J2000 frame, respectively; $\boldsymbol{g}_{\mathrm{mI}}$ is the gravity vector in the J2000 inertial frame, which can be calculated according to the lander position and Mars gravity field model contained in the Mars environment module; $\boldsymbol{a}_{\mathrm{thr}}, \boldsymbol{a}_{\mathrm{atm}}, \boldsymbol{a}_{\mathrm{para}}$, and $\boldsymbol{a}_{\text {hea }}$ denote the accelerations caused by the engine thrust, aerodynamic force, parachute drag force, and recoil force of heatshield ejection, respectively; $\boldsymbol{q}$ is the lander attitude quaternion describing the rotation from the J2000 inertial frame to the lander body frame; $\boldsymbol{\omega}$ is the angular velocity corresponding to the lander attitude; $\boldsymbol{I}$ is the inertial matrix of the lander; and $\boldsymbol{T}_{\mathrm{thr}}, \boldsymbol{T}_{\mathrm{atm}}, \boldsymbol{T}_{\mathrm{para}}$, and $\boldsymbol{T}_{\text {hea }}$ are the torques associated with the engine thrust, aerodynamic force, parachute drag force, and recoil force of heatshield ejection, respectively. The accelerations $\boldsymbol{a}_{\mathrm{thr}}, \boldsymbol{a}_{\mathrm{atm}}, \boldsymbol{a}_{\mathrm{para}}$, and $\boldsymbol{a}_{\text {hea }}$ are calculated as

$$
\left\{\begin{array}{l}
\boldsymbol{a}_{\mathrm{thr}}=\frac{\boldsymbol{F}_{\mathrm{thr}}}{m}=\sum_{i=0}^{n} \frac{\boldsymbol{F}_{\mathrm{thr} i}}{m} \\
\boldsymbol{a}_{\text {atm }}=\frac{\boldsymbol{F}_{\text {atm }}}{m} \\
\boldsymbol{a}_{\text {para }}=\frac{\boldsymbol{F}_{\text {para }}}{m} \\
\boldsymbol{a}_{\text {hea }}=\frac{\boldsymbol{F}_{\text {hea }}}{m}
\end{array}\right.
$$

where $m$ is the lander mass with the consumption rate $\dot{m}=\sum_{i=0}^{n} \frac{\left\|\boldsymbol{F}_{\mathrm{thr} i}\right\|}{I_{\mathrm{sp} i}} ; n$ is the thrust number; $\boldsymbol{F}_{\mathrm{thr} i}$ and $I_{\mathrm{sp} i}$ are the thrust and impulse of the $i$ th thruster, respectively; $\boldsymbol{F}_{\text {atm }}, \boldsymbol{F}_{\text {para }}$, and $\boldsymbol{F}_{\text {hea }}$ are the aerodynamic force, parachute drag force, and recoil force of heatshield ejection, respectively.

To solve the dynamics described in Eqs. (1) and (2), the forces (i.e., $\boldsymbol{F}_{\text {thri }}, \boldsymbol{F}_{\text {atm }}, \boldsymbol{F}_{\text {para }}$, and $\boldsymbol{F}_{\text {hea }}$ ) and torques (i.e., $\boldsymbol{T}_{\text {thr }}, \boldsymbol{T}_{\text {atm }}, \boldsymbol{T}_{\text {para }}$, and $\boldsymbol{T}_{\text {hea }}$ ) must be calculated. Using the thrust model, the engine force, $\boldsymbol{F}_{\text {thri }}$, can be calculated according to the thruster pulse width commands controlled by the GNC module. Torques $\boldsymbol{T}_{\text {para }}$ and $\boldsymbol{T}_{\text {hea }}$ are calculated using the parachute dynamics model and heatshield dynamics model, respectively. In particular, $\boldsymbol{F}_{\text {para }}=\mathbf{0}$ and $\boldsymbol{T}_{\text {para }}=\mathbf{0}$ in the atmospheric entry phase and landing phase, and $\boldsymbol{F}_{\text {para }}$ and $\boldsymbol{T}_{\text {para }}$ have to be solved only in the parachute descent phase for the lander, as described in the following section. The variation of $\boldsymbol{F}_{\text {hea }}$ and $\boldsymbol{T}_{\text {hea }}$ with time occurs over a period of only $100 \mathrm{~ms}$; they are modeled using the heatshield dynamics module. For the entire EDL phase, $\boldsymbol{F}_{\text {atm }}$ and $\boldsymbol{T}_{\text {atm }}$ are calculated and modeled as follows:

$$
\left\{\begin{aligned}
\boldsymbol{F}_{\mathrm{atm}}= & \boldsymbol{C}_{\mathrm{q}}^{\mathrm{T}}\left[\begin{array}{l}
C_{\mathrm{A}} \\
C_{\mathrm{N}} \\
C_{\mathrm{Z}}
\end{array}\right] q_{\mathrm{p}} S_{\mathrm{ref}} \\
\boldsymbol{T}_{\mathrm{atm}}= & \boldsymbol{C}_{\mathrm{q}}^{\mathrm{T}}\left[\begin{array}{l}
C_{\mathrm{T} x} \\
C_{\mathrm{T} y} \\
C_{\mathrm{T} z}
\end{array}\right] q_{\mathrm{p}} S_{\mathrm{ref}} D_{\mathrm{ref}} \\
& +\boldsymbol{C}_{\mathrm{q}}^{\mathrm{T}}\left[\begin{array}{ccc}
0 & 0 & 0 \\
0 & C_{\mathrm{dT} y} & 0 \\
0 & 0 & C_{\mathrm{dT} z}
\end{array}\right] \frac{\omega}{v_{\mathrm{r}}} q_{\mathrm{p}} S_{\mathrm{ref}} D_{\mathrm{ref}}^{2}
\end{aligned}\right.
$$

where $C_{\mathrm{q}}$ is the rotation matrix from the body frame to the J2000 frame; $q_{\mathrm{p}}=\frac{1}{2} \rho v_{\mathrm{r}}^{2}$ is the aerodynamic pressure; $\rho$ is the atmospheric density at a current position (provided by the Mars atmosphere model); $v_{\mathrm{r}}$ is the airspeed magnitude calculated as the difference in the lander velocity relative to the Mars surface and wind speed; $D_{\text {ref }}$ is the reference diameter with $S_{\text {ref }}=$ $\frac{\pi D_{\text {ref }}^{2}}{4} ; C_{\mathrm{A}}, C_{\mathrm{N}}$, and $C_{\mathrm{Z}}$ are the axial, normal, and side aerodynamic force coefficients, respectively; $C_{\mathrm{T} x}, C_{\mathrm{T} y}$, and $C_{\mathrm{T} z}$ are the roll, pitch, and yaw moment coefficients, respectively; and $C_{\mathrm{dT} y}$ and $C_{\mathrm{dT} z}$ are the pitch and yaw dynamic derivative coefficients, respectively.

The aerodynamic force, moment, and dynamic derivative coefficients can be calculated by interpolating the corresponding aerodynamic coefficient databases [12]. To support the EDL phase, several aerodynamic coefficient databases are modeled according to different phases and configurations based on empirical or 
simulation data. When the altitude exceeds $44.8 \mathrm{~km}$, the aerodynamic force and moment coefficients (known as static aerodynamic coefficients) are modeled for a specific range of altitudes and total attack angles. When the altitude is less than $44.8 \mathrm{~km}$, and the trim wing is not deployed, the static aerodynamic coefficient is modeled for different Mach numbers and total attack angles. In the trim wing deployment process, the static aerodynamic coefficient is modeled according to the deployed angles of the trim wing and total attack angles. After trim wing deployment, the static aerodynamic coefficient is modeled considering the Mach numbers, attack angles, and sideslip angles. In particular, during the heatshield and backshell separation process, the separating object moves extremely close to the lander, and the two entities exert considerable aerodynamic effects on each other. Therefore, the static aerodynamic coefficient is modeled considering the Mach numbers, attack angles, sideslip angles, and distances between the lander and separating object. The aerodynamic derivative coefficients are modeled considering the Mach numbers, attack angles, and sideslip angles, accounting for the cases of trim wing deployment and nondeployment.

Using these aerodynamic coefficient databases, the orbit and attitude dynamics can be simulated throughout the Mars EDL phase, especially operations. Because of the uncertainty in aerodynamics, aerodynamic coefficient bias databases are constructed in the simulator. These are inconsistent with the corresponding coefficient databases and are used in the Monte Carlo analysis, as described in the next section.

\subsection{Atmosphere and wind modeling}

Atmospheric and wind modeling is performed to specify the atmospheric temperature, density, and wind velocity calculated by interpolating the Mars atmospheric environment databases according to the altitude in the simulator. The databases contain nominal values and their bias ranges. The nominal values and bounds of density, temperature, and horizontal wind speed are shown in Figs. 4-6, constructed with reference to the Mars Science Laboratory (MSL). The vertical wind speed is assumed to be zero when the altitude exceeds $20 \mathrm{~km}$ and set as a zero mean value with a $20 \mathrm{~m} / \mathrm{s}$ deviation when the altitude is less than $20 \mathrm{~km}$ [9]. These bounds represent the uncertainties in the atmosphere, which are

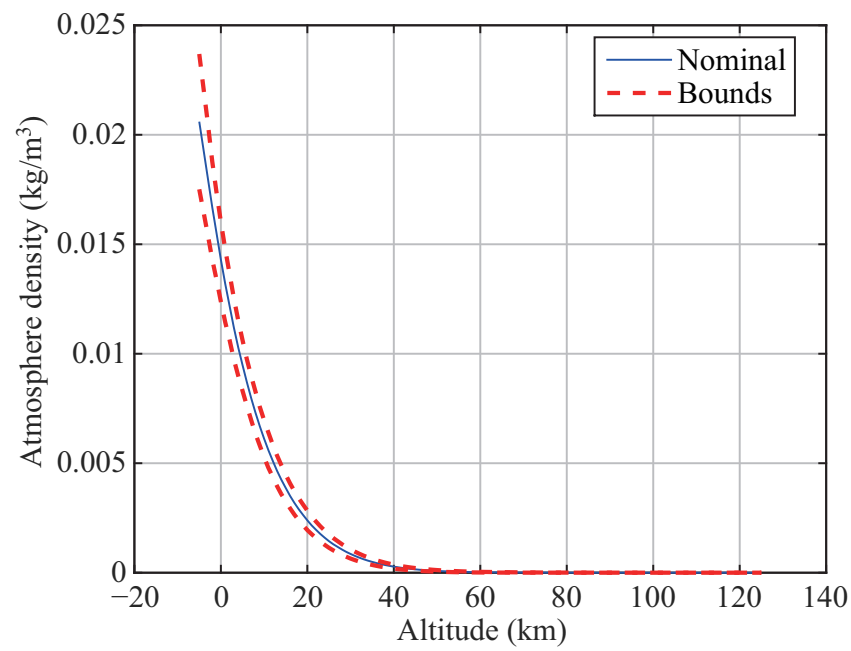

Fig. 4 Nominal atmosphere density and its bounds at different altitudes.

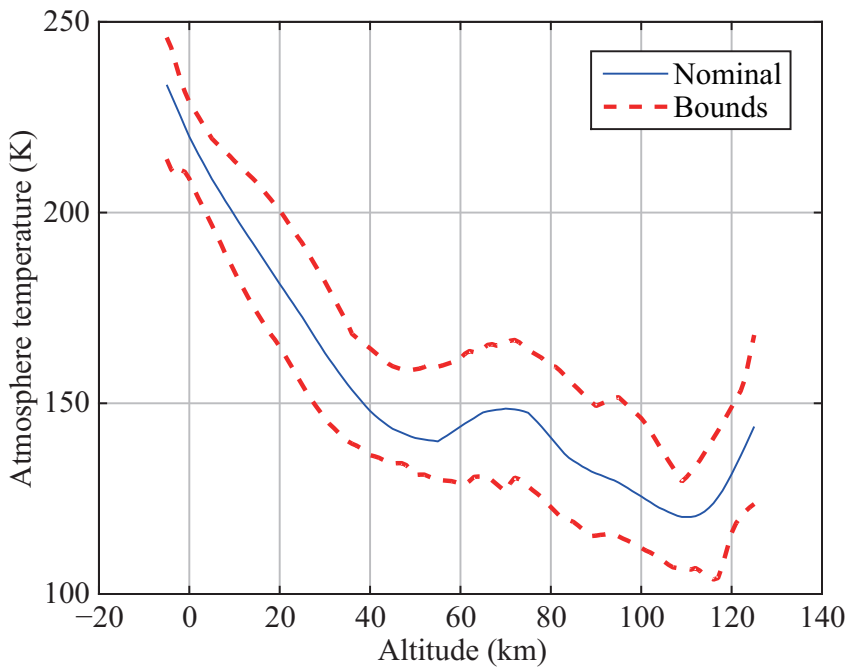

Fig. 5 Nominal atmosphere temperature and its bounds at different altitudes.

considered in the Monte Carlo analysis described in the next section.

After determining the atmospheric temperature, density, and wind velocity, the Mach number, aerodynamic pressure, and angles of attack and sideslip can be calculated. These values are inputted to the orbit and attitude dynamics model to solve the aerodynamic force and torque.

\subsection{Parachute dynamics and modeling}

The Tianwen-1 lander parachute is similar to that of the MSL described in Ref. [13] and contains a single riser and four bridles. The parachute is connected to the backshell by the four bridles, and the parachute drag 


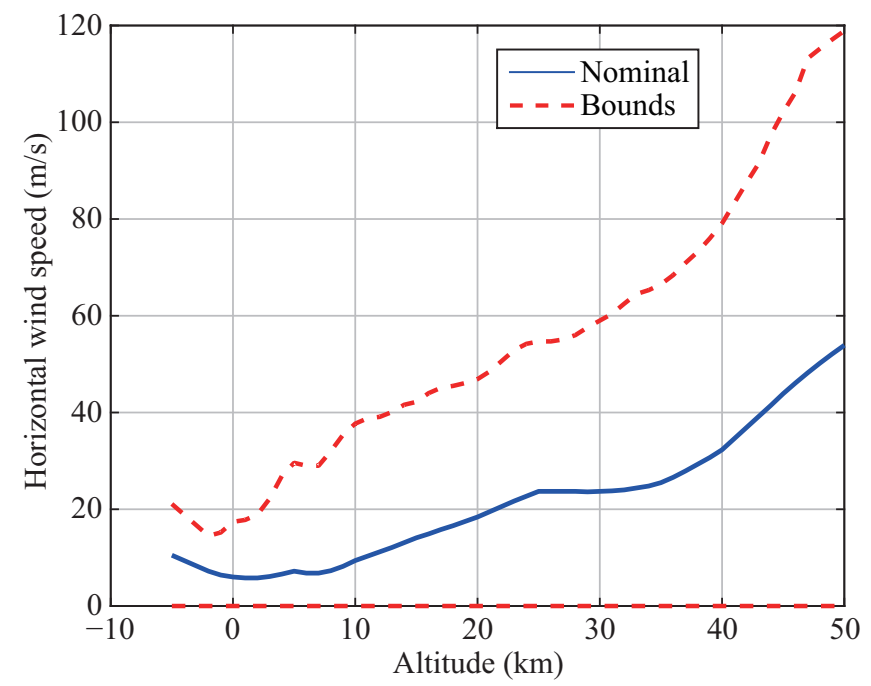

Fig. 6 Nominal horizontal wind speed and its bounds at different altitudes.

force is calculated according to the change in the length of each bridle.

Parachute descent consists of the following phases: parachute ejection, parachute line stretching, parachute inflation, and stabilized descent. Before the parachute lines stretch, solving the parachute dynamics is unnecessary, and only the parachute ejection force must be considered because the recoil force of ejection is imposed on the lander. The parachute ejection phase lasts from mortar firing at time $t_{0}$ to the parachute bag reaching the mortar muzzle velocity at time $t_{\text {open }}$. In the parachute ejection phase, the mortar ejection force is set as $100 \mathrm{kN}$ for Tianwen-1. Assuming that the parachute lines stretch at time $t_{\mathrm{ls}}$, and backshell separation occurs at time $t_{\mathrm{sepB}}$, the parachute drag force, $\boldsymbol{F}_{\text {para }}$, on the lander (Eq. (2)) can be calculated. This force is estimated according to the change in the length of each bridle, which can be determined from the poses of the parachute and backshell. After calculating $\boldsymbol{F}_{\text {para }}$, the corresponding torque, $\boldsymbol{T}_{\text {para }}$, on the lander can be calculated according to the position of the connecting point between the bridles and backshell.

After the parachute lines stretch, the parachute dynamics are modeled similar to that of the Tianwen-1 lander. In the model, the forces on the parachute are gravity, aerodynamic force $\left(\boldsymbol{F}_{\text {atmP }}\right)$, and parachute drag recoil force $\left(\boldsymbol{F}_{\text {paraP }}\right)$. The recoil force is calculated as

$$
\boldsymbol{F}_{\text {paraP }}=-\boldsymbol{F}_{\text {para }}
$$

and the corresponding torque, $\boldsymbol{T}_{\mathrm{paraP}}$, on the parachute can be calculated according to the position of the connecting point between the bridles and riser.
The aerodynamic force $\left(\boldsymbol{F}_{\text {atmP }}\right)$ and torque $\left(\boldsymbol{T}_{\text {atmP }}\right)$ acting on the parachute are modeled as

$$
\left\{\begin{aligned}
\boldsymbol{F}_{\mathrm{atmP}}= & \boldsymbol{C}_{\mathrm{qP}}^{\mathrm{T}}\left(\boldsymbol{e}_{\mathrm{x}} C_{\mathrm{AP}}+\boldsymbol{e}_{\mathrm{N}} C_{\mathrm{NP}}\right) K_{\mathrm{KI}} K_{\mathrm{AOM}} K_{\mathrm{MEF}} \\
& \cdot K_{\mathrm{Off}} q_{\mathrm{pP}} S_{\mathrm{refP}} \\
\boldsymbol{T}_{\mathrm{atmP}}= & \boldsymbol{C}_{\mathrm{qP}}^{\mathrm{T}} \boldsymbol{e}_{\mathrm{T}} C_{\mathrm{TP}} K_{\mathrm{KI}} K_{\mathrm{AOM}} K_{\mathrm{MEF}} K_{\mathrm{Off}} \\
& \cdot q_{\mathrm{pP}} S_{\mathrm{refP}} D_{\mathrm{refP}}
\end{aligned}\right.
$$

where $\boldsymbol{C}_{\mathrm{qP}}$ is the rotation matrix from the parachute body frame to the J2000 frame; $q_{\mathrm{pP}}$ is the dynamic pressure acting on the parachute; $D_{\text {refP }}$ is the reference diameter with $S_{\mathrm{refP}}=\frac{\pi D_{\mathrm{refP}}^{2}}{4} ; K_{\mathrm{KI}}, K_{\mathrm{AOM}}, K_{\mathrm{MEF}}$, and $K_{\text {Off }}$ denote the inflation factor, area oscillation parameter, Mach efficiency factor, and offloading model parameter, respectively (details are found in Ref. [13]); and $C_{\mathrm{AP}}, C_{\mathrm{NP}}$, and $C_{\mathrm{TP}}$ are the axial and normal aerodynamic force coefficients and pitch moment coefficient, respectively, describing the static aerodynamic coefficients of the parachute because the parachute is axisymmetric.

The static aerodynamic coefficients of the parachute, $C_{\mathrm{AP}}, C_{\mathrm{NP}}$, and $C_{\mathrm{TP}}$, can be calculated by interpolating the corresponding parachute aerodynamic coefficient databases in our simulator; they are modeled for the total angle of attack, as shown in Figs. 7-9. The parachute dynamics solution step must not exceed $1 \mathrm{~ms}$ due to the considerable dynamic oscillation of the parachute.

\subsection{Heatshield and backshell dynamics modeling}

The heatshield or backshell dynamics are solved when the objects separate from the landing platform, and the

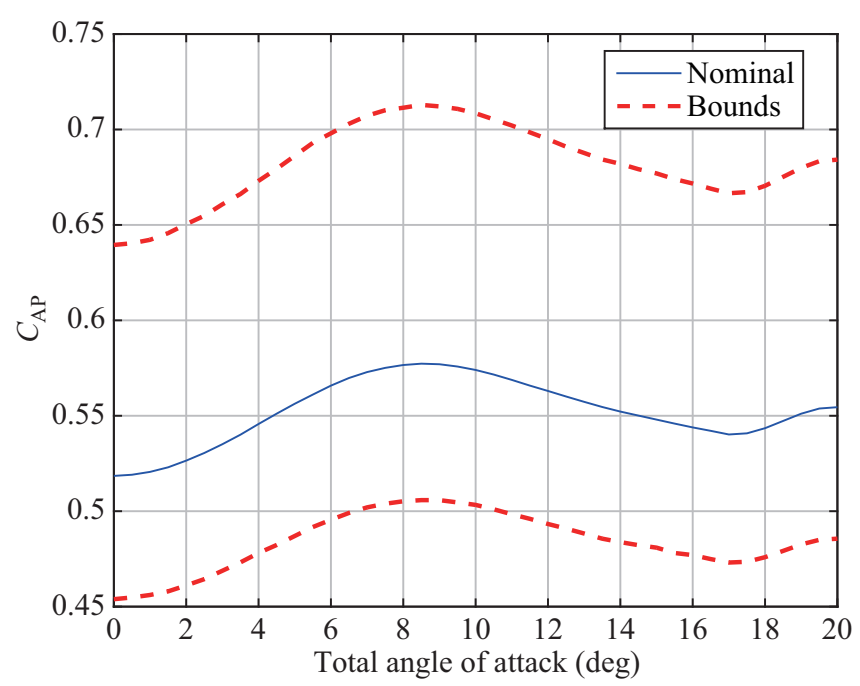

Fig. 7 Axis aerodynamic force coefficient of Tianwen-1 parachute. 


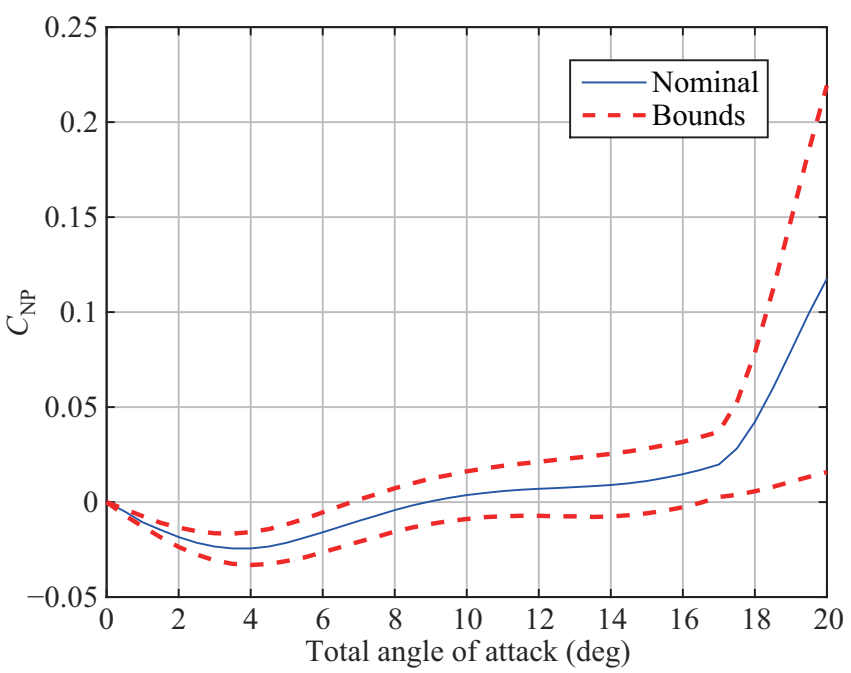

Fig. 8 Normal aerodynamic force coefficient of Tianwen-1 parachute.

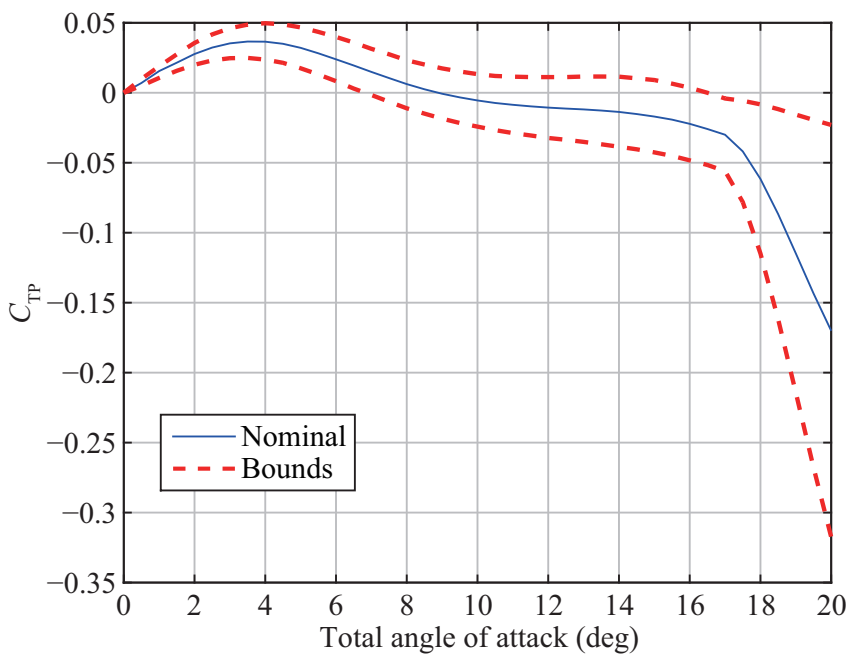

Fig. 9 Pitch aerodynamic moment coefficient of Tianwen-1 parachute.

model of the orbit and attitude dynamics is similar to that of the lander described in Eq. (1). In the dynamics model, the forces acting on the heatshield are gravity and aerodynamic force; the forces acting on the backshell are gravity, aerodynamic force, and parachute drag force. The aerodynamic force is calculated by interpolating the corresponding aerodynamic coefficients of the simulator, and the parachute drag force is calculated according to the change in the length of each bridle, as described in Eq. (5). However, the heatshield is ejected through the explosion of four bolts, and the explosive force acting on each bolt is imposed on both heatshield and landing platform. The total explosive force impact on the heatshield is defined as $\boldsymbol{F}_{\text {heaH }}=-\boldsymbol{F}_{\text {hea }}=\sum_{i=0}^{4} \boldsymbol{F}_{\text {heaH } i}$ over a period of only $100 \mathrm{~ms}$. The explosive force direction is along the $x$ axis of the lander, and its magnitude is modeled as a function of the distance between the heatshield and landing platform, as shown in Fig. 10. The torque generated by the explosive force can be calculated according to the installation location of the explosive bolts.

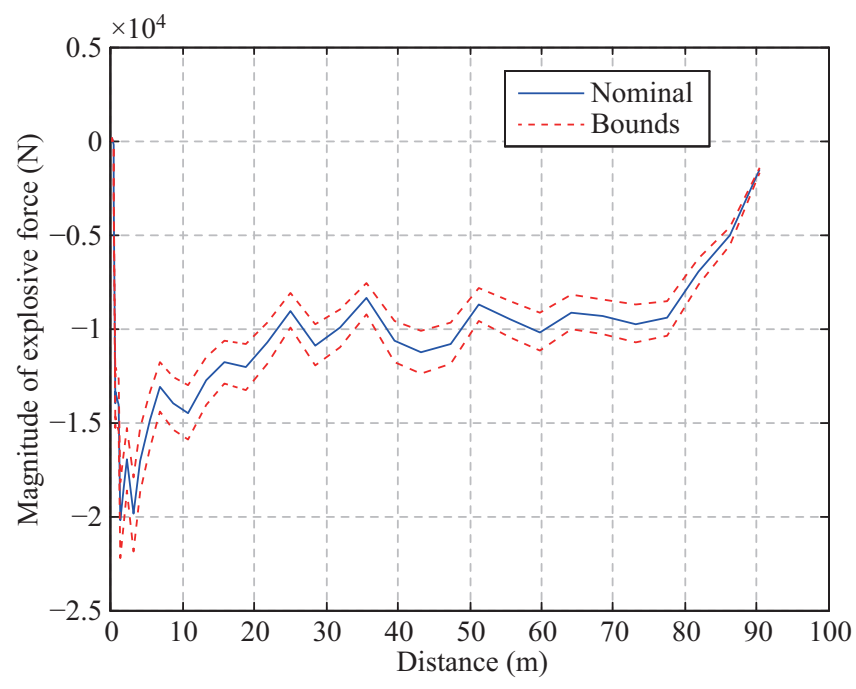

Fig. 10 Magnitude of explosive force at different distances between heatshield and landing platform.

\subsection{GNC module for Tianwen-1 EDL}

The GNC module contains eight GNC modes: attack angle trim mode, lift control mode, parachute descent control mode, powered deceleration mode, hover and imaging mode, hazard avoidance maneuver mode, slow descent mode, and no-control mode. In each mode, the GNC module processes data from sensors, performs real-time GNC calculations, and transmits the control signals for orbit and attitude controls. The details of the Tianwen-1 lander GNC algorithm are presented in Ref. [3].

\section{$5 \quad$ Performance metrics}

\subsection{Mars EDL simulation results under nominal conditions}

This section describes the simulation under nominal conditions. The initial states of the lander at the atmosphere interface are set as those used in the actual flight operation on May 15, 2021, as listed in Table 1. The initial position and velocity are expressed in the Mars-centered J2000 frame. 
Table 1 Initial states of Tianwen-1 lander EDL

\begin{tabular}{ll}
\hline State & Values \\
\hline Initial time (BJT) & May 15, 2021 at 7:8:54.7233 \\
Initial position $(\mathrm{m})$ & 3163492.266471, \\
& -1522037.547769, \\
& 147228.268912 \\
Initial velocity & $-215.964964069,1333.853595255$, \\
$\quad(\mathrm{m} / \mathrm{s})$ & -4605.398643736 \\
Initial attitude & -0.039209842429785279, \\
$\quad$ quaternion & 0.58049005131366760, \\
& 0.23268682873675028, \\
& 0.77932709969237013 \\
Initial mass $(\mathrm{kg})$ & 1272.34 \\
\hline
\end{tabular}

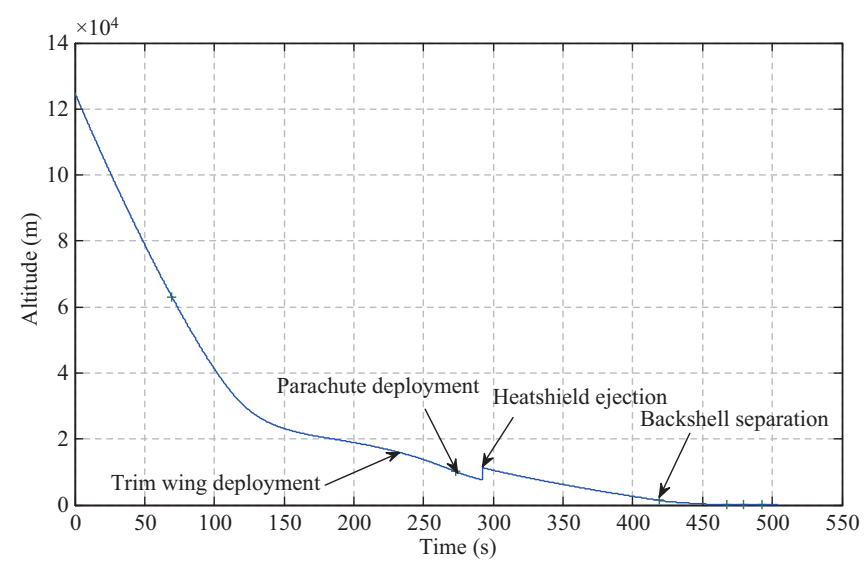

Fig. 11 Simulation of actual altitude during Mars EDL by Tianwen-1 simulator.
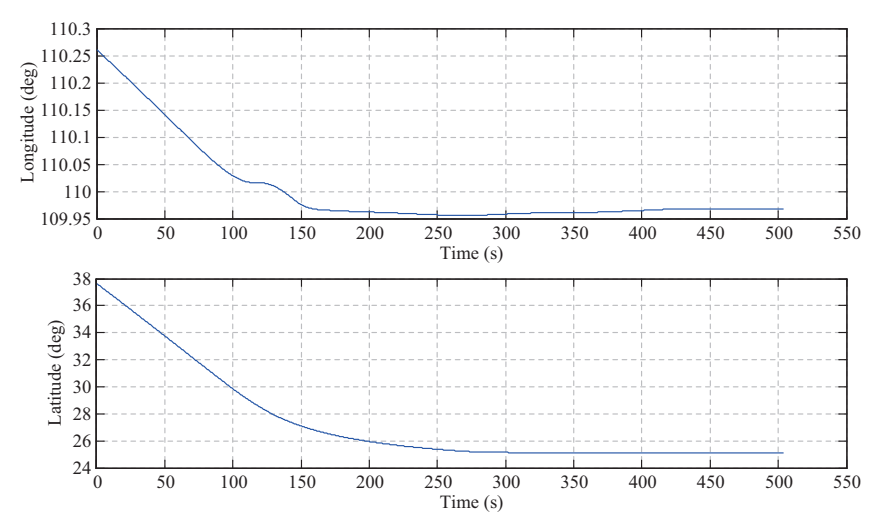

Fig. 12 Simulation of actual longitude and latitude during Mars EDL.

In the simulation of the nominal case, the uncertainties in the atmospheric parameters and aerodynamic coefficients described in Section 4 are not considered. The dynamic states of the lander simulated during the EDL phase by the constructed simulator are shown in Figs. 11-14. The trim wing is deployed at an altitude of $15.8 \mathrm{~km}$ when the Mach number is 2.8 . The parachute
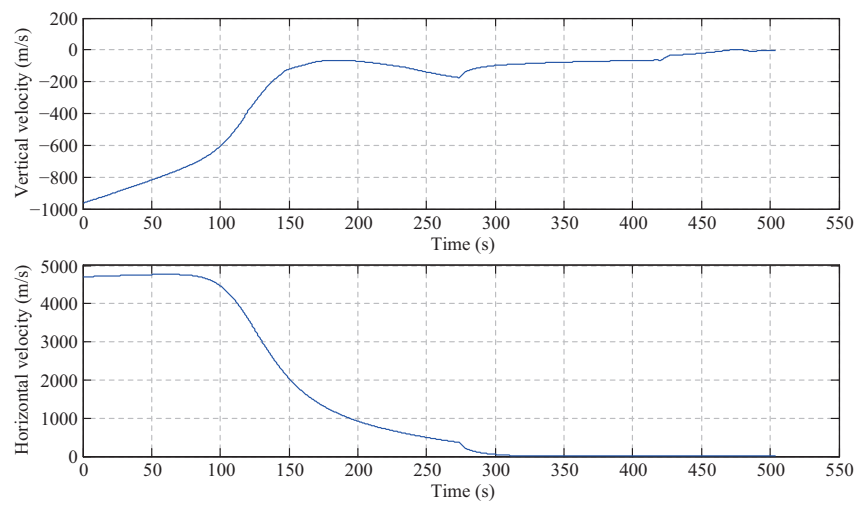

Fig. 13 Simulation of vertical and horizontal velocities during Mars EDL.

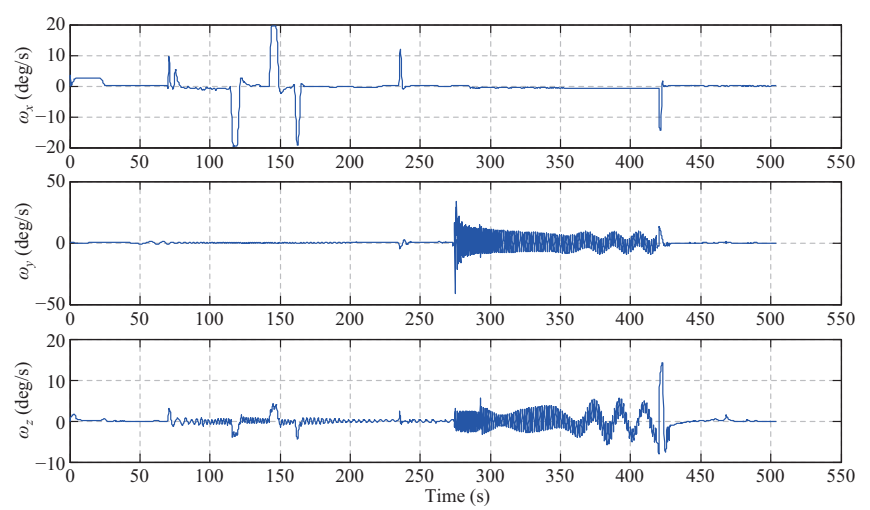

Fig. 14 Simulation of angular velocity during Mars EDL.

is deployed at an altitude of $10.1 \mathrm{~km}$ when the Mach number is 1.8. The heatshield is ejected at an altitude of $11.32 \mathrm{~km}$, and the backshell is separated at an altitude of $1.4 \mathrm{~km}$ with vertical and horizontal velocities of -63.65 and $8.17 \mathrm{~m} / \mathrm{s}$, respectively. The altitude exhibits a jump change when the heatshield is ejected because the terrain model is incorporated in the simulation, and the altitude is relative to the local terrain elevation instead of the Mars ellipsoid surface. At surface touchdown, the vertical and horizontal velocities are -1.54 and $0.19 \mathrm{~m} / \mathrm{s}$, respectively. The angular velocity is less than $0.1^{\circ}$, which satisfies the requirements of safe and soft landing. In this simulation, note that the duration of the parachute descent phase is between 273 and $420 \mathrm{~s}$. During this period, parachute inflation and area oscillation can cause high angular rate oscillation. Meanwhile, rate-damping controllers are used for the pitch and yaw channels with long deadbands, and the angle and angle rate error are controlled for the roll channel. As a result, the pitch and yaw angle rates oscillate during the parachute descent phase, as shown in Fig. 14.

The Mach number, angles of attack and sideslip, and 


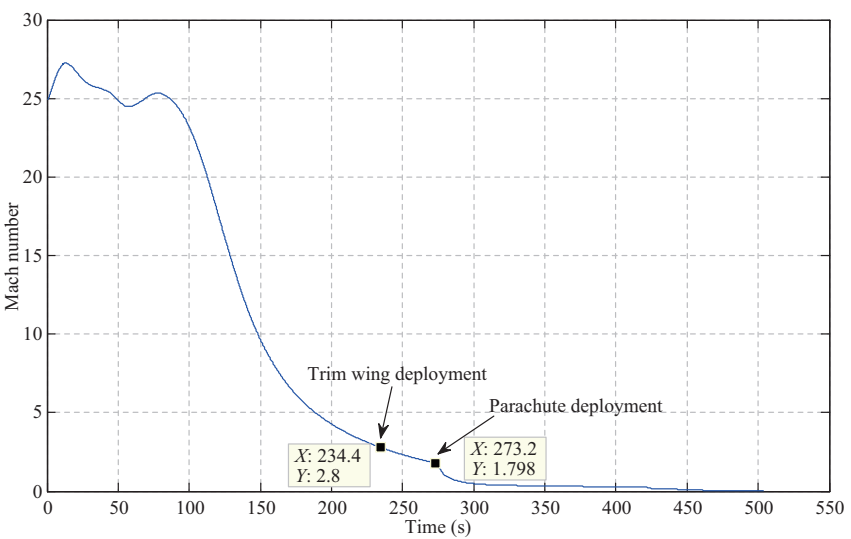

Fig. 15 Simulation of Mach number during Mars EDL.
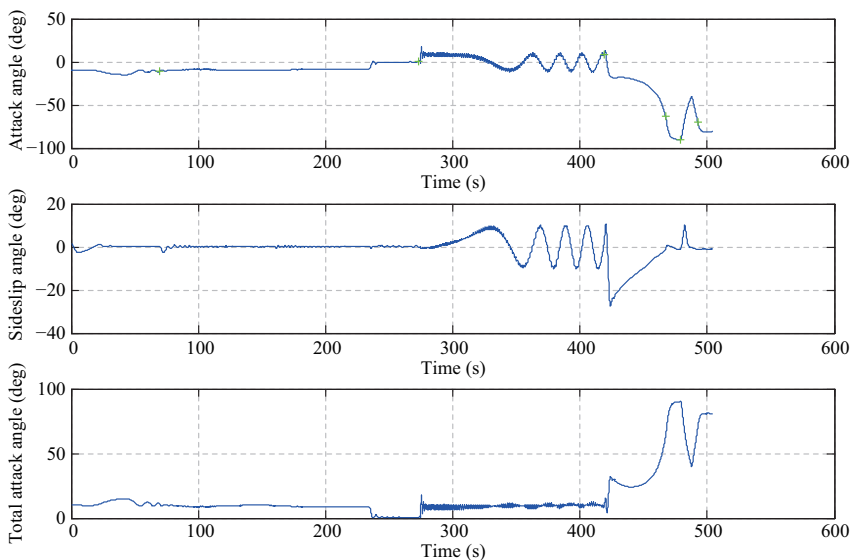

Fig. 16 Simulation of angles of attack and sideslip, and total attack angle during Mars EDL.
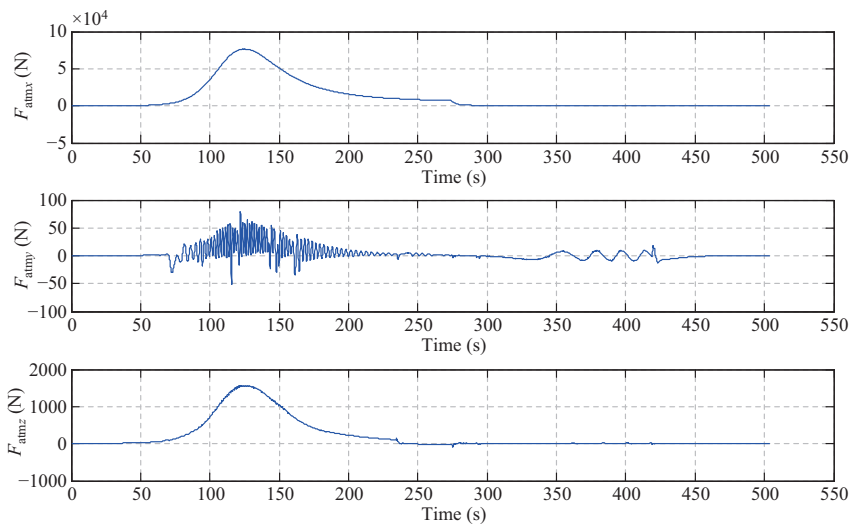

Fig. 17 Simulation of aerodynamic force impact on lander body frame during Mars EDL.

total attack angle of the lander during EDL that are used to calculate the aerodynamic coefficients are shown in Figs. 15 and 16. The aerodynamic force, parachute drag force, and their corresponding torque impact on the lander are shown in Figs. 17-20. As a result of construction modifications associated with heatshield
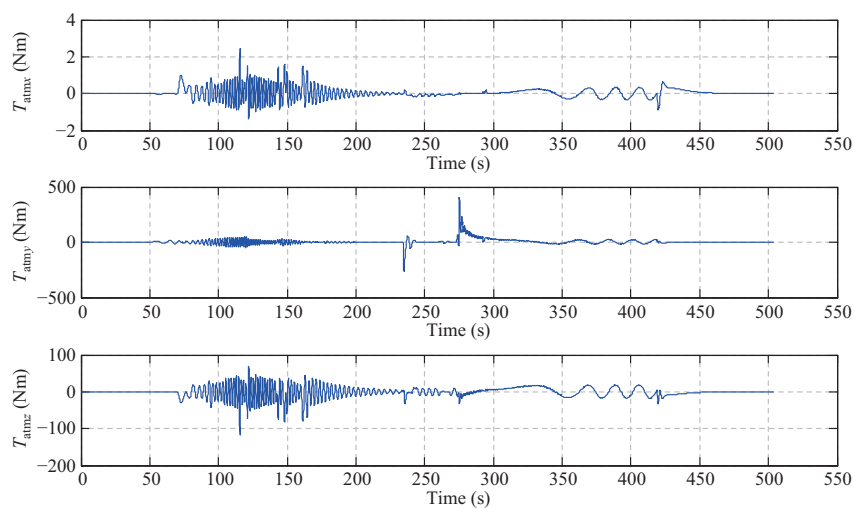

Fig. 18 Simulation of aerodynamic torque impact on lander body frame during Mars EDL.
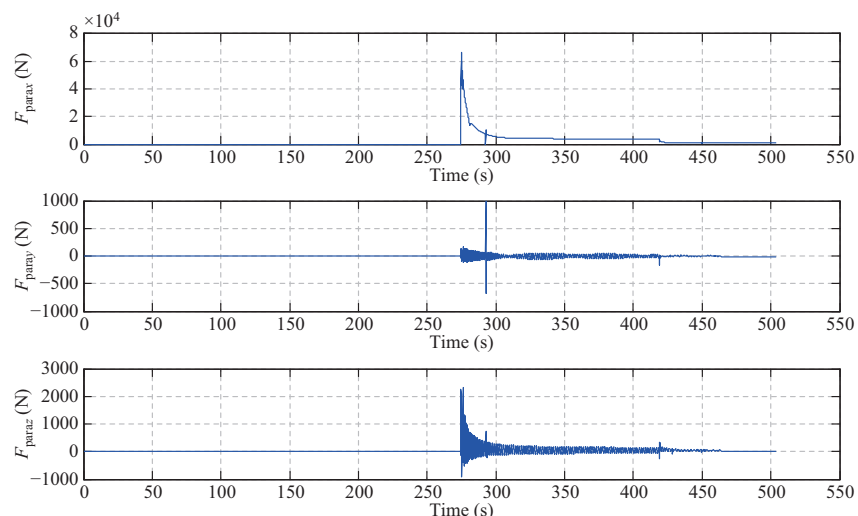

Fig. 19 Simulation of parachute drag force impact on lander during Mars EDL.
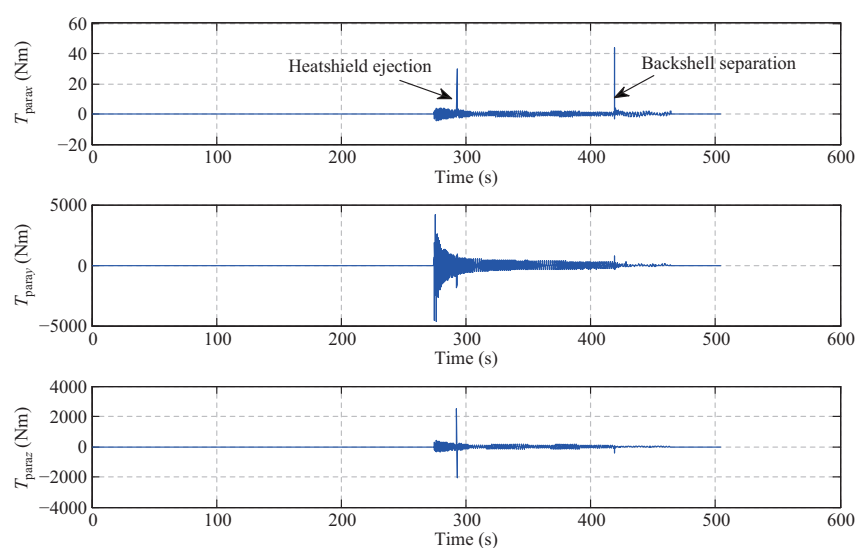

Fig. 20 Simulation of parachute drag torque impact on lander during Mars EDL.

ejection and backshell separation, the parachute drag torque exhibits two jump changes at two points, as shown in Fig. 20. All simulated dynamics conform with the forecast, and reliable results are obtained.

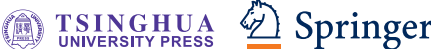




\subsection{Monte Carlo analysis}

To assess the performance and robustness of the GNC scheme designed for the Tianwen-1 lander, a Monte Carlo analysis is performed using the constructed simulator. The analysis is implemented considering the uncertainties in the key parameters and coefficients of the environment and lander as well as the uncertainties in the initial states of the lander. The uncertainties in the atmospheric parameters and aerodynamic coefficients are described in Section 4, and the uncertainties of the initial states and characteristics of the lander are listed in Table 2 (specified as $3 \sigma$ or $\mathrm{min} / \max$ values dispersed from the nominal values). The uncertainties in position and velocity are expressed in the orbit coordinate system, in which the $x y$ plane coincides with the orbit plane, the $x$ axis is along the radial direction, the $y$ axis points to the velocity direction, and the $z$ axis is along the normal direction of the orbit plane. The uncertainties in the sensors and actuators are also included in the simulation; however, they are not presented in this paper.

Table 2 Uncertainties in initial state and characteristics of Tianwen-1 lander

\begin{tabular}{ll}
\hline State uncertainty & $3 \sigma$ or min $/$ max value \\
\hline Initial position $(\mathrm{m})$ & $6500,15000,2500$ \\
Initial velocity $(\mathrm{m} / \mathrm{s})$ & $12.0,2.5,3.0$ \\
Initial attitude $\left(^{\circ}\right)$ & $\pm 0.1, \pm 0.1, \pm 0.1$ \\
Initial mass $(\mathrm{kg})$ & \pm 10.0 \\
Inertial moments & $\pm 10 \%, \pm 10 \%, \pm 10 \%$, \\
$\quad I_{x x}, I_{y y}, I_{z z}$ & respectively \\
Inertial moments & $\pm 5 \%, \pm 5 \%, \pm 10 \%$, \\
$\quad I_{x y}, I_{x z}, I_{y z}$ & respectively \\
Initial position of center & $\pm 0.03, \pm 0.005, \pm 0.005$ \\
$\quad$ & \\
\hline
\end{tabular}

The 10,000-iteration Monte Carlo simulation results are shown in Figs. 21-30. The figures present several key states of the lander at the trim wing deployment (Figs. 21 and 22), parachute deployment (Figs. 23 and 24), and surface touchdown (Figs. 25-30). The constraints of corresponding states, which ensure that the trim wing and parachute are successfully deployed and that the soft landing is safe, are also shown. According to the constraints, the Mach number lies within $2.8 \pm 0.45$, the aerodynamic pressure lies between 500 and $1200 \mathrm{~Pa}$, the attack angle lies within $-10^{\circ} \pm 8.5^{\circ}$, and the sideslip angle is within $0^{\circ} \pm 5^{\circ}$. At trim wing deployment, the Mach number lies within $1.8 \pm 0.3$, the aerodynamic

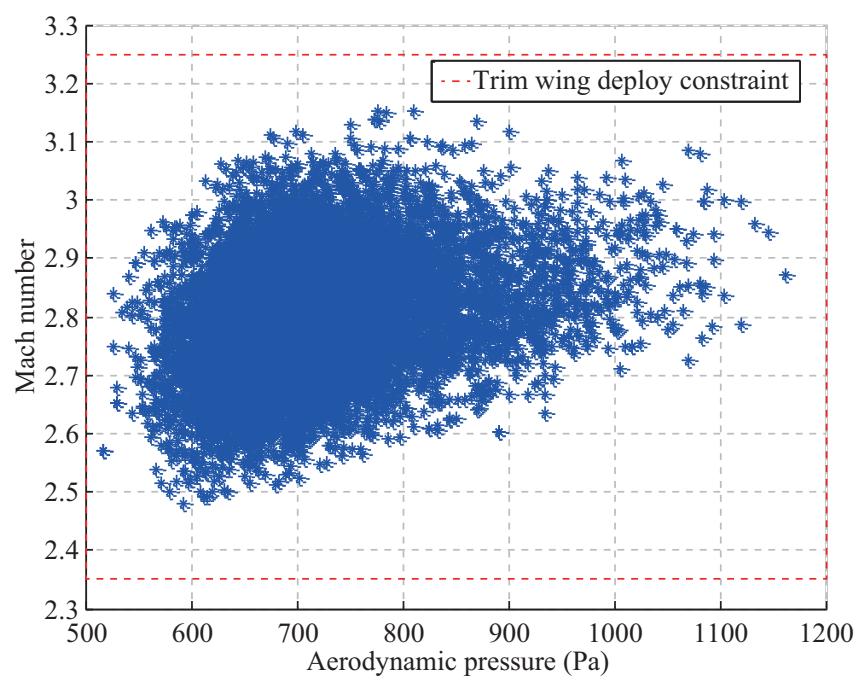

Fig. 21 Mach number and aerodynamic pressure during trim wing deployment.

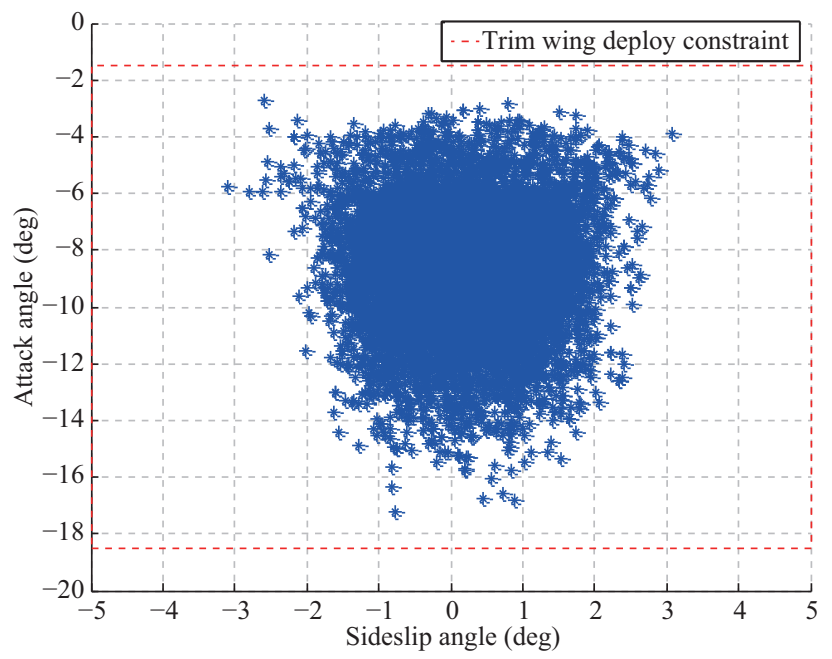

Fig. 22 Angles of attack and sideslip during trim wing deployment.

pressure is within $560 \pm 200 \mathrm{~Pa}$, and the altitude is between 4 and $15 \mathrm{~km}$. At parachute deployment, the vertical velocity lies within $-1.5 \pm 0.6 \mathrm{~m} / \mathrm{s}$, the horizontal velocity is less than $0.9 \mathrm{~m} / \mathrm{s}$, the angular velocity is less than $1\left(^{\circ}\right) / \mathrm{s}$, and the angle between the $x$ axis and vertical direction is less than $2.5^{\circ}$ at touchdown. The Monte Carlo simulation results indicate that the constraints are satisfied in all 10,000 iterations.

In addition, the distributions of the propellant consumption and duration of EDL are examined to analyze the landing mission safety. The results shown in Figs. 29 and 30 indicate that the propellant consumption is $112-130 \mathrm{~kg}$, and the duration is within $410-650 \mathrm{~s}$. The position and velocity navigation errors at touchdown, 


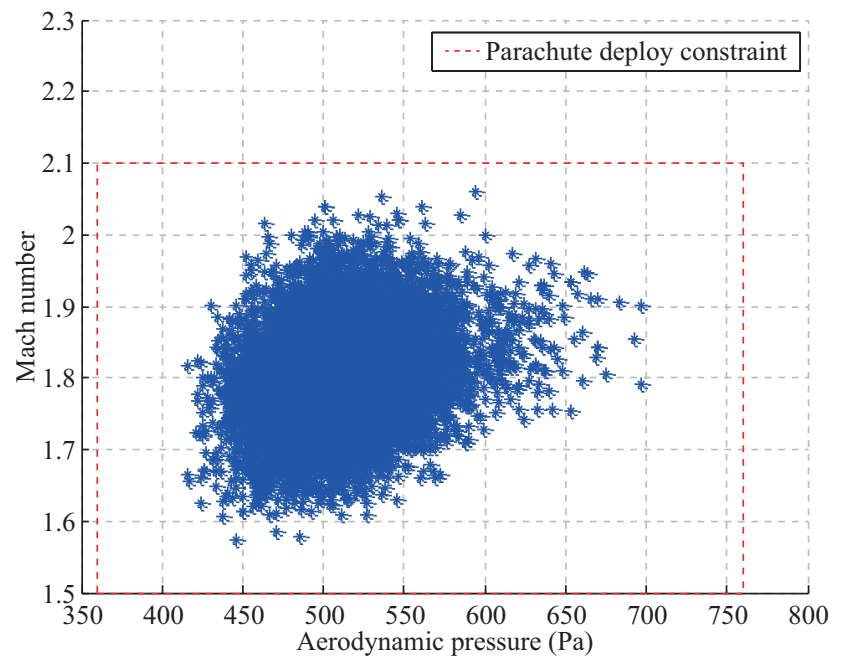

Fig. 23 Mach number and aerodynamic pressure during parachute deployment.

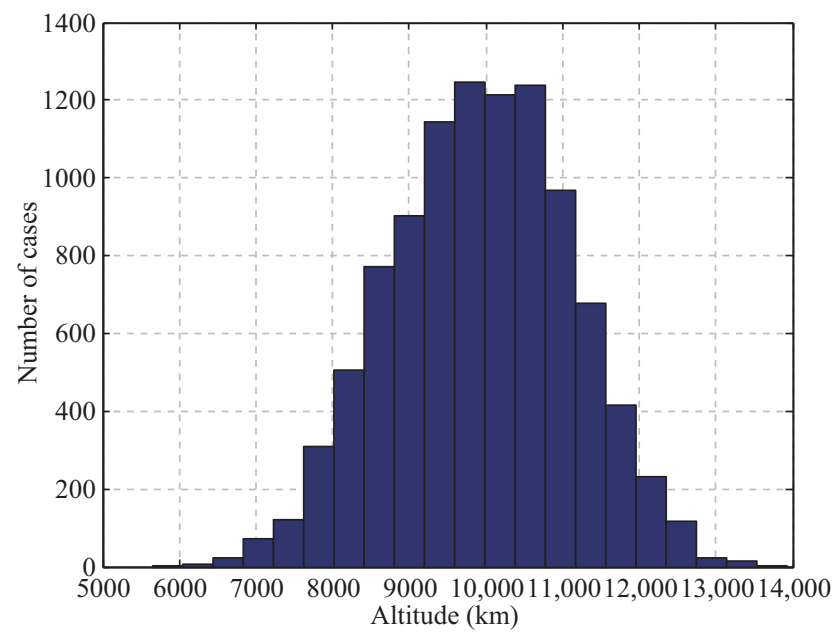

Fig. 24 Altitude distribution during parachute deployment.

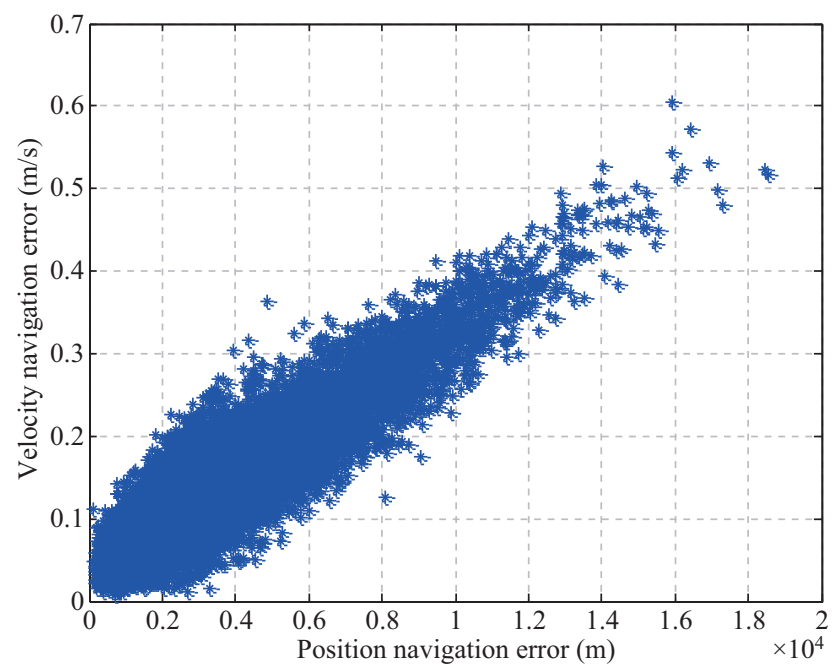

Fig. 25 Position and velocity navigation errors at touchdown.

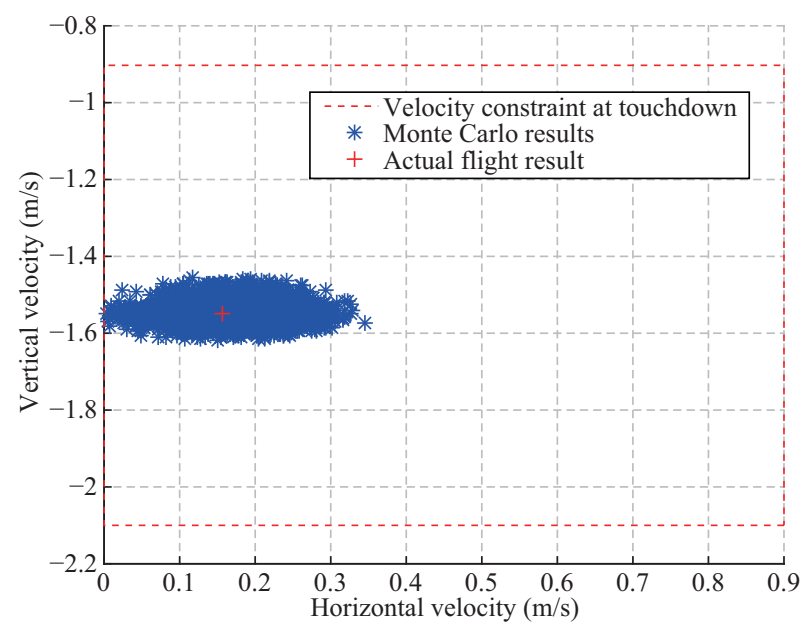

Fig. 26 Vertical and horizontal velocities at touchdown.

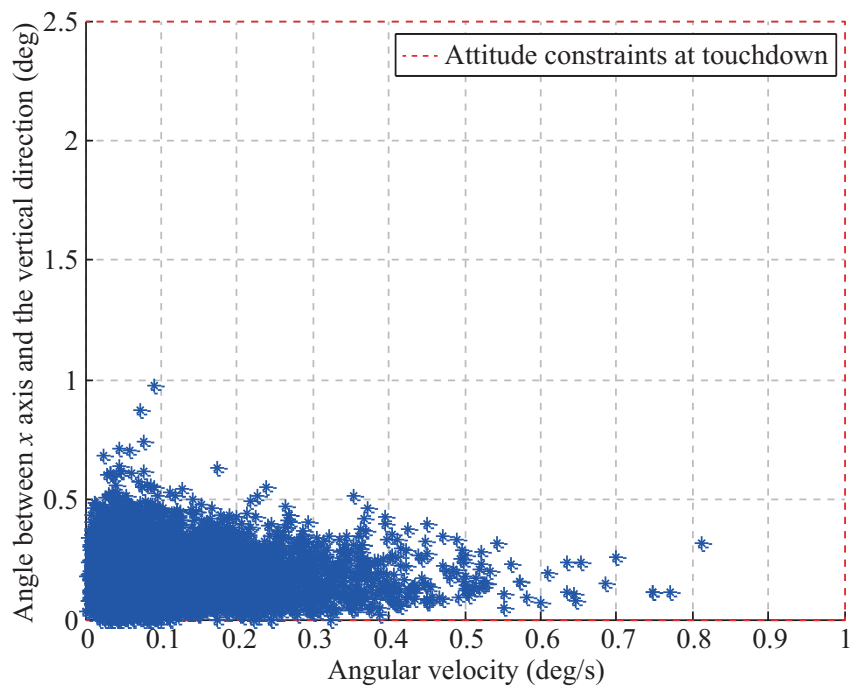

Fig. 27 Angular velocity and angle between $x$ axis and vertical direction at touchdown.

which are less than $18 \mathrm{~km}$ and $0.55 \mathrm{~m} / \mathrm{s}$, respectively, are shown in Fig. 25. The main component of the position navigation error is the horizontal position error because the altitude and velocity are corrected by the radar sensor. The simulated landing sites in 10,000 runs and corresponding landing ellipses as well as the target and actual landing sites of the Tianwen-1 lander are shown in Fig. 28. The results shown in this figure indicate that the actual flight of the Tianwen- 1 lander is within the envelope of the Monte Carlo simulation.

The Monte Carlo simulation generates a considerable amount of lander information during EDL that can be used to fully assess the performance of the GNC system. Not all information is presented here because of limited paper length.

\section{(10) UNIINGSITY TUA}




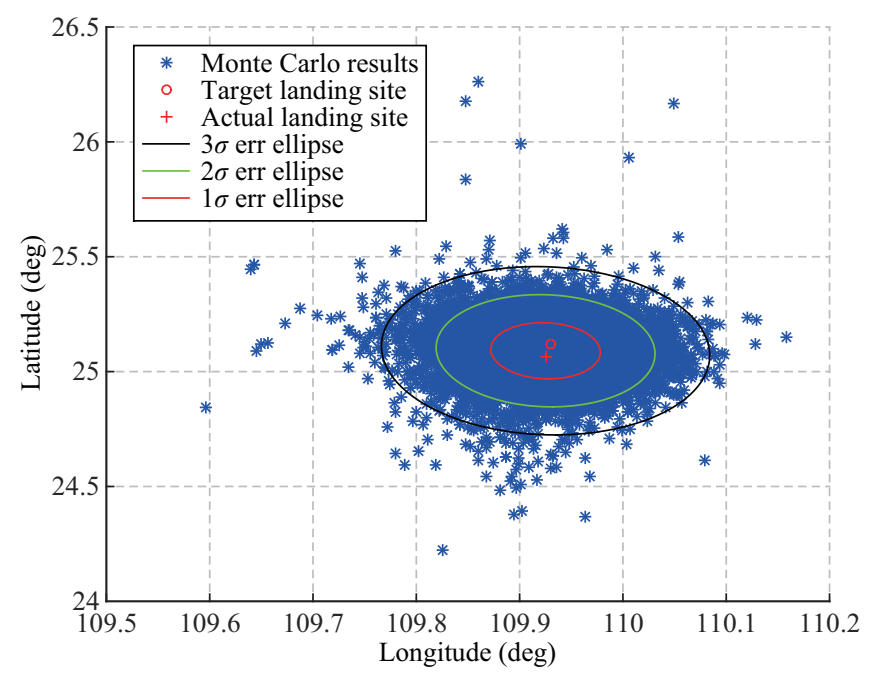

Fig. 28 Landing site.

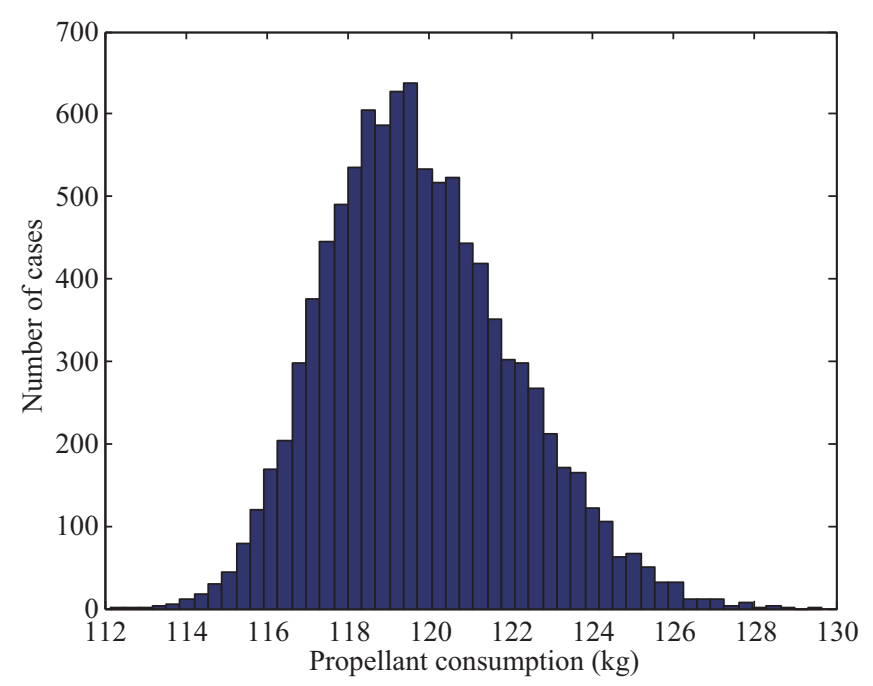

Fig. 29 Propellant consumption distribution in EDL phase.

\section{Conclusions}

An end-to-end Mars EDL simulator for the Tianwen-1 GNC system is developed. The multibody dynamic models for the lander, parachute, backshell, and heatshield in the simulator are described. Using the developed simulator, simulation under nominal conditions and Monte Carlo statistical analysis are performed to assess the performance and robustness of the Tianwen-1 lander GNC system. The simulation results indicate that the simulator is valid, the Tianwen-1 lander GNC system for the Mars EDL exhibits high performance, and the key parameters in the EDL phase satisfy the requirements. The simulator plays a key role in the development of the Tianwen-1 lander GNC system and can be applied to the next generation of Mars exploration systems.

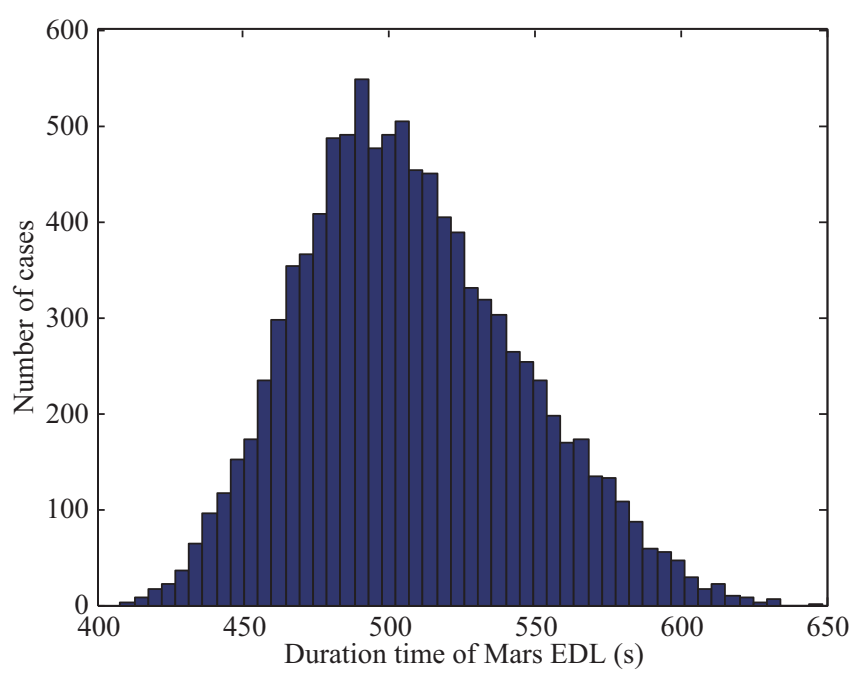

Fig. 30 Distribution of EDL duration.

\section{Acknowledgements}

This work was supported by the National Natural Science Foundation of China (No. 61803028) and Civil Aerospace Advance Research Project.

\section{Conflict of interest}

The authors declare that there is no conflict of interest.

\section{References}

[1] Desai, P. N., Prince, J. L., Queen, E. M., Schoenenberger, M. M., Cruz, J. R., Grover, M. R. Entry, descent, and landing performance of the Mars phoenix lander. Journal of Spacecraft and Rockets, 2011, 48(5): 798-808.

[2] Karlgaard, C. D., Korzun, A. M., Schoenenberger, M., Bonfiglio, E. P., Kass, D. M., Grover, M. R. Mars InSight entry, descent, and landing trajectory and atmosphere reconstruction. Journal of Spacecraft and Rockets, 2021, 58(3): 865-878.

[3] Huang, X. Y., Li, M. D., Wang, X. L., Hu, J. C., Zhao, Y., Guo, M. W., Xu, C., Liu, W. W., Wang, Y. P., Hao, C., Xu, L. J. The Tianwen-1 guidance, navigation, and control for Mars entry, descent, and Landing. Space: Science and Technology, 2021, 2021: 9846185.

[4] Queen, E., M., Prince, J. L., Desai, P. N. Multibody modeling and simulation for Mars Phoenix entry, descent, and landing. Journal of Spacecraft and Rockets, 2011, 48(5): 766-771.

[5] Brauer, G. L., Cornick, D. E., Stevenson, R. Capabilities and applications of the program to optimize simulated trajectories (POST). NASA CR-2770, 1977.

[6] Behzad, R., Eric, Q., Nathaniel, J. H. Validation of multibody program to optimize simulated trajectories 
II parachute simulation with interacting forces. NASA TP-2009-215765, 2009.

[7] Cameron, J. M., Jain, A., Burkhart, P. D., Bailey, E. S., Balaram, B., Bonfiglio, E., Ivanov, M., Benito, J., Sklyanskiy, E., Strauss, W. DSENDS: Multi-mission flight dynamics simulator for NASA missions. In: Proceedings of AIAA SPACE, 2016: 5421-5438.

[8] Balaram, J., Austin, R., Banerjee, P., Bentley, T., Henriquez, D., Martin, B., McMahon, E., Sohl, G. DSENDS-A high-fidelity dynamics and spacecraft simulator for entry, descent and surface landing. In: Proceedings of the IEEE Aerospace Conference, 2002, 7: 3343-3359.

[9] Prince, J. L., Desai, P. N., Queen, E. M., Grover, M. R. Mars phoenix entry, descent, and landing simulation design and modeling analysis. Journal of Spacecraft and Rockets, 2011, 48(5): 756-764.

[10] Striepe, S. A., Way, D. W., Dwyer, A. M., Balaram, J. Mars science laboratory simulations for entry, descent, and landing. Journal of Spacecraft and Rockets, 2006, 43(2): 311-323.

[11] Chen, A., Vasavada, A., Cianciolo, A., Barnes, J., Tyler, D., Rafkin, S., Hinson, D., Lewis, S. Atmospheric risk assessment for the Mars Science Laboratory Entry, Descent, and Landing system. In: Proceedings of the IEEE Aerospace Conference, 2010: 1-12.

[12] Edquist, K. T., Desai, P. N., Schoenenberger, M. Aerodynamics for Mars phoenix entry capsule. Journal of Spacecraft and Rockets, 2011, 48(5): 713-726.

[13] Cruz, J. R., Way, D., Shidner, J., Davis, J. L., Powell, R. W., Kipp, D., Adams, D. S., Sengupta, A., Witkowski, A., Kandis, M. Parachute models used in the Mars science laboratory entry, descent, and landing simulation. In: Proceedings of the AIAA Aerodynamic Decelerator Systems Conference, 2013: AIAA 2013-1276.

[14] Cui, P. Y., Qin, T., Zhu, S. Y. Progress in autonomous navigation and guidance technology for Mars powered descent landing. Journal of Astronautics, 2020, 41(1): 1-9. (in Chinese).

[15] Huang, X. Y., Xu, C., Hu, R. H., Li, M. D., Guo, M. W., Hu, J. C. Research of autonomous navigation and control scheme based on multi-information fusion for Mars pinpoint landing. Journal of Deep Space Exploration, 2019, 6(4): 348-357. (in Chinese)

[16] Gao, X. L., Zhang, Q. B., Tang, Q. G. Numerical modelling of Mars supersonic disk-gap-band parachute inflation. Advances in Space Research, 2016, 57(11): 2259-2272.

[17] Lu, Y. Y., Rong, W., Wu, S. T. Dynamic modeling of parachute deployment in Mars environment. Spacecraft Recovery 85 Remote Sensing, 2014, 35(1): 29-36.
[18] Zhang, Q. B., Feng, Z. W., Ma, Y., Ge, J. Q., Cao, X. L., Cao, Q. Y. Modeling and simulation of Mars EDL process. Journal of Astronautics, 2017, 38(5): 443-450. (in Chinese)

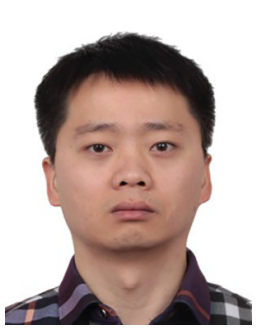

Chao Xu received his B.S. degree from Beihang University, China, in 2010 and then received his M.E. and Ph.D. degrees in guidance, navigation, and control from China Academy of Space Technology (CAST), China, in 2013 and 2017, respectively. $\mathrm{He}$ is currently working as an engineer at Beijing Institute of Control Engineering (BICE), China. His research interests include autonomous navigation, vision-aided navigation, and simultaneous localization and mapping. E-mail: xc_1987@ 126.com.

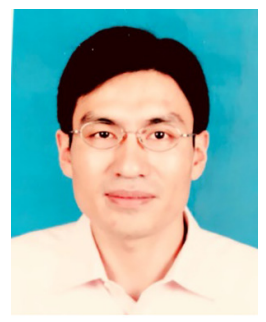

Xiangyu Huang received his B.S. and Ph.D. degrees in aerospace engineering from Harbin Institute of Technology, China, in 1999 and 2005, respectively. Since August 2005, he has been with Science and Technology on Space Intelligent Control Laboratory of Beijing Institute of Control Engineering (BICE), where he is currently a professor and senior research specialist. He was a guidance, navigation, and control (GNC) engineer of the Chang'e-3 lunar landing mission, and his current research area is GNC design for planetary landing missions. He won one State Technological Invention Award (second class) and three Ministerial and Provincial-level Science and Technology Awards (first class). E-mail: huangxyhit@sina.com.

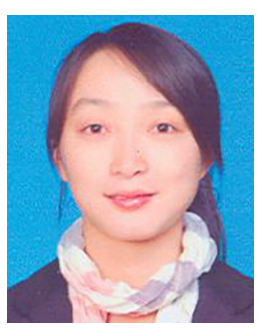

Minwen Guo received her Ph.D. degree in guidance, navigation, and control from China Academy of Space Technology in 2014. Then, she worked as an engineer at Beijing Institute of Control Engineering. From 2016 to 2021, she participated in the Mars Tianwen-1 development and was engaged in the design of the Martian atmosphere entry guidance law. In 2019, she was certified as a senior engineer. Currently, her research interests are spacecraft entry guidance and trajectory optimization. E-mail: mwguo8500@163.com.

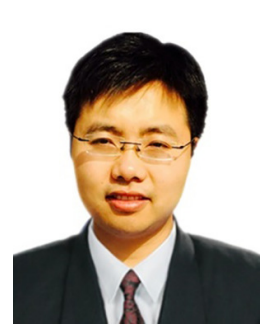

Maodeng Li received his B.S. and Ph.D. degrees in aerospace engineering from Harbin Institute of Technology, China, in 2006 and 2011, respectively. From September 2011 to August 2013, he was a postdoctoral research associate at BICE. Since August 2013, he has been with Science and Technology on Space 
Intelligent Control Laboratory of BICE as a senior engineer. His current research areas include spacecraft autonomous navigation and GNC design for planetary landing missions. E-mail: mdeng1985@gmail.com.

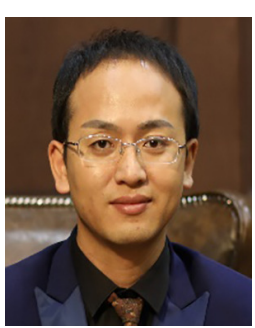

Jinchang Hu received his B.S., M.E., and Ph.D. degrees from Beihang University, Tsinghua University, and Beijing Institute of Control Engineering, respectively. He is currently a senior engineer at Beijing Institute of Control Engineering. His main research interests include spacecraft attitude and orbit control. E-mail: hujinchang@tsinghua.org.cn.

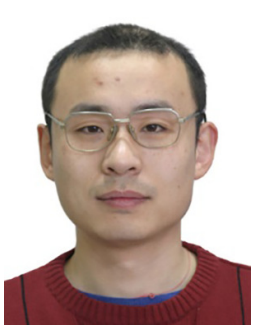

Xiaolei Wang is currently a professor at Beijing Institute of Control Engineering. His main research interest lies in spacecraft GNC system design.
Open Access This article is licensed under a Creative Commons Attribution 4.0 International License, which permits use, sharing, adaptation, distribution and reproduction in any medium or format, as long as you give appropriate credit to the original author(s) and the source, provide a link to the Creative Commons licence, and indicate if changes were made.

The images or other third party material in this article are included in the article's Creative Commons licence, unless indicated otherwise in a credit line to the material. If material is not included in the article's Creative Commons licence and your intended use is not permitted by statutory regulation or exceeds the permitted use, you will need to obtain permission directly from the copyright holder.

To view a copy of this licence, visit http://creativecomm ons.org/licenses/by/4.0/. 